\title{
Utilização do Anel de Ferrara na estabilização e correção da ectasia corneana pós PRK
}

\author{
Use of Ferrara's ring in the stabilization and correction of corneal ectasia after PRK
}

Frederico Bicalho Dias da Silva ${ }^{(1)}$ Eduardo Adan França Alves (2) Paulo Ferrara de Almeida Cunha ${ }^{(3)}$

\section{RESUMO}

Objetivo: Avaliar a ação do anel corneano intra-estromal de Ferrara no tratamento da ectasia corneana pós Excimer Laser.

Métodos: $O$ anel de Ferrara, que já vem sendo aplicado no tratamento do ceratocone, alta miopia e astigmatismo irregular, foi utilizado para estabilizar e corrigir a ectasia corneana de dois pacientes submetidos à PRK.

Resultados: Após o procedimento cirúrgico, foi observado um aplainamento da córnea central de aproximadamente 6 dioptrias no $1^{0}$ caso e de 10 dioptrias no $2^{\circ}$, com melhora na acuidade visual. Os resultados vêm se mantendo estáveis até o momento. $O$ tempo de seguimento pós-operatório foi de 3 e 8 meses, respectivamente.

Conclusões: Anel de Ferrara obteve sucesso no difícil tratamento desta complicação cirúrgica, permitindo que se retarde ou até mesmo se evite a evolução destes casos para um transplante de córnea com todos os seus riscos, lenta recuperação e dependência da agilidade das filas de doações.

Palavras-chave: Ectasia; Cirurgia refrativa; Anel de Ferrara.

\section{INTRODUÇÃO}

Há mais de 15 anos, o excimer laser entrou para o mundo oftalmológico e iniciou uma verdadeira revolução. Iniciaram, na década de 80 , estudos utilizando essa nova tecnologia para cirurgias corneanas com finalidade curativa ("phototherapeutic keratectomy", ou PTK) e refrativa ("photorefractive keratectomy", ou PRK). Durante esses estudos, aprendeu-se que o PRK não era adequado para correção da alta miopia ${ }^{5}$, o que culminou com o desenvolvimento de uma técnica híbrida entre a keratomileusis convencional e o próprio PRK, chamada de LASIK ("laser in situ keratomileusis"). De fato, a ablação excessiva da córnea gera complicações que variam desde o "haze" até a ectasia ${ }^{4}$. Esta última provoca o retorno da miopia de forma progressiva sendo paliativamente controlada com o uso de óculos ou lentes de contato, podendo ainda evoluir para a ceratoplastia penetrante como solução definitiva, levando-se em conta todos os riscos e problemas inerentes a este procedimento.

Em 1986, foi desenvolvido o anel corneano intraestromal (ACI) chamado Anel de Ferrara que passou a ser utilizado para a correção de ceratocone, alta miopia e astigmatismo irregular. Neste trabalho, mostraremos uma nova aplicação do Anel de Ferrara: a estabilização e correção da ectasia corneana após a ceratectomia fotorefrativa. 


\section{MATERIAL E MÉTODO}

\section{Caso 1}

Trata-se de J.A.C., 49 anos, masculino, mecânico, residente em Contagem - MG.

Portador de alta miopia em OE tratada cirurgicamente com PRK em dezembro de 1995, tendo como exame pré-operatório:

Refração: $\mathrm{OD}=$ plano $(\mathrm{AV}=20 / 20) \quad \mathrm{OE}=-12,5 \mathrm{D}(\mathrm{AV}=20 / 40)$

Ceratometria: $\mathrm{OD}=44,50 \mathrm{D} \times 44,50 \mathrm{D} \quad \mathrm{OE}=44,50 \mathrm{D} \times 44,50 \mathrm{D}$

Evoluiu com regressão do resultado refracional e "haze", estando em abril de 1996 com 5,50D esféricas negativas no OE e acuidade visual corrigida de 20/100. A biomicroscopia mostrava a existência de "haze" $3+/ 4+$. Foi então submetido a nova sessão de PRK.

Em agosto de 1996, apresentava novamente "haze" 3+/4+ e acuidade visual de 20/100 (refração do $\mathrm{OE}=$ plano). Optouse por nova sessão de PRK seguida da utilização de corticosteróide tópico na forma de colírio (dexametasona $0,1 \%$ ) cinco vezes ao dia.

Foi então encaminhado até o nosso serviço em setembro de 1997, com o seguinte exame:

Refração: $\mathrm{OD}=$ plano (AV=20/20) $\mathrm{OE}=-6,00$ esf. $(\mathrm{AV}=20 / 200)$

A biomicroscopia mostrava a presença de "haze" $4+/ 4+$ em OE. Foi iniciado o uso de colírio de Oncotiotepa 0,06\% 4 vezes ao dia.

Em outubro de 1997, permanecia com AV cc $=20 / 200$ e "haze" 4+/4+, sendo então realizada escarificação da córnea do OE com lâmina de bisturi $n^{\circ} 15$ e PRK, visando correção de $-1,00 \mathrm{D}$ e polimento. Duas semanas após, encontrava-se com $-0,50 \mathrm{D}$ esf. e AV $=20 / 50+$.

Já em janeiro de 1998, teve nova regressão do estado refracional $(\mathrm{AV} \mathrm{cc}=20 / 200)$ e aumento do haze $(4+/ 4+)$, sendo feito fotoablação para polimento da córnea com equipamento NIDEK.

Em novembro de 1998, encontrava-se com o seguinte exame:

Refração: $\mathrm{OE}=-7,25 \mathrm{D}(\mathrm{AV}=20 / 80)$

Biomicroscopia: $\mathrm{OE}=$ "haze" 1+/4+

Paquimetria computadorizada $($ ORBSCAN $)$ central $=259$ micra

Uma vez reduzido o "haze", restava a correção da ectasia corneana. Assim, em novembro de 1998, optamos pelo implante do Anel de Ferrara. Trata-se de dois segmentos de anel de PMMA de secção transversal triangular, diâmetro de ápice de $6 \mathrm{~mm}$ e comprimento de $150^{\circ}$. Em cada extremidade destes segmentos existe um orifício que permite sua manipulação durante o procedimento de implantação. A técnica cirúrgica consistiu na utilização de instrumento que possui dupla lâmina semicircular, sendo capaz de confeccionar de maneira fácil e simultânea os dois túneis corneanos estromais. Nestes, fo- ram introduzidos os dois segmentos de anel citados anteriormente. A cirurgia foi realizada sob anestesia tópica. No pósoperatório, foi prescrito FML 4 vezes ao dia durante o primeiro mês.

\section{Caso 2}

E. A., 33 anos, masculino, comerciante, com diagnóstico de ceratocone há 5 anos. Foi submetido, bilateralmente, a tratamento com PRK há 3 meses. Embora tenha obtido um resultado satisfatório a princípio, iniciou rapidamente com lenta e progressiva piora da $\mathrm{AV}$ acompanhado de miopização de ambos os olhos. Foi então encaminhado a este serviço com o seguinte exame:

AVcc: $\mathrm{OD}=20 / 30(-14,00)-\mathrm{OE}=20 / 40(-16,00)$

Ceratometria: $\mathrm{OD}>52,00 \mathrm{Dx}>52,00 \mathrm{D}-\mathrm{OE}>52,00 \mathrm{Dx}>52,00 \mathrm{D}$

Biomicroscopia: córnea afilada AO com opacidades endoteliais.

Biomicroscopia ultra-sônica: Espessura corneana no ápice do cone: $\mathrm{OD}=365 \mu \mathrm{m}-\mathrm{OE}=365 \mu \mathrm{m}$

\section{Cirurgia:}

Em 17/08/98, foi submetido a implante de Anel de Ferrara no OE (AF5-60-150) (diâmetro de ápice $=5 \mathrm{~mm}$; largura da base $=60$ micra; comprimento de $\operatorname{arco}=150^{\circ}$ )

\section{RESULTADOS}

\section{Caso 1}

Na próxima página estão as topografias do pré- e do pósoperatório do implante do Anel de Ferrara, respectivamente, figuras 1 e 2 .

Seu último retorno ao Centro de Oftalmologia Avançada ocorreu no dia 26 de fevereiro de 1999, após 3 meses do implante do ACI, tendo o seguinte exame:

Ceratometria: 38,03D x 34,23D (astig.:3,80D). Refração: $+1,50-2,50 \times 90^{\circ}(\mathrm{AV}=20 / 40)$.

Biomicroscopia: Conjuntivas calmas, segmentos de anel bem implantados no estroma médio, câmara anterior sem células ou "flare". Não ocorreu alteração na transparência corneana central, permanecendo o pequeno "haze" de 1+/4+ do pré-operatório.

\section{Caso 2}

Na próxima página estão as topografias do pré- e do pósoperatório do implante do Anel de Ferrara, respectivamente, figuras 3 e 4 .

Em 18/08/98 (1º dia pós-op), encontrava-se com AV do OE $s c=20 / 50$, mas o segmento temporal do anel sofreu um deslocamento superior de forma que sua extremidade alcançava a incisão. Este quadro foi acompanhado até o dia 27/08/98, quando se optou pelo reposicionamento do segmento temporal.

Em 30/08/98 (13 dias pós-op), estava com o seguinte exame: 


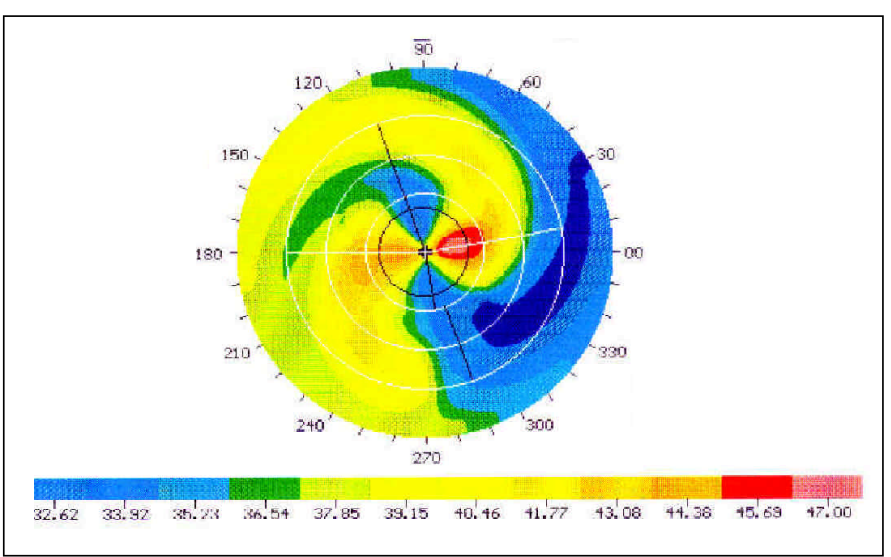

Fig. 1 - Aspecto topográfico do pré-operatório do caso 1 em 28/11/98.

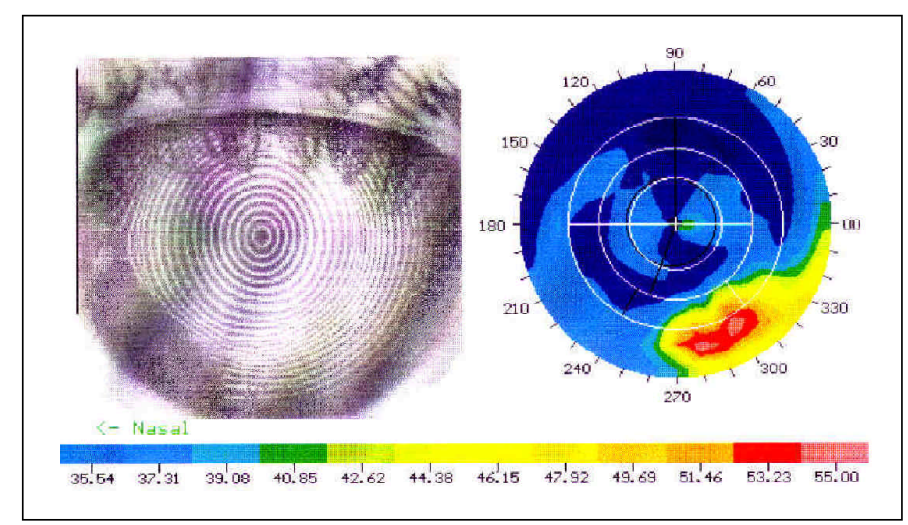

Fig. 2 - Aspecto topográfico do pós-operatório do caso 1 em 26/02/99.

AVsc: $\mathrm{OE}=20 / 40$

Ceratometria: $37,00 \mathrm{D}$ x 37,00D com miras mais ou menos regulares.

Biomicroscopia: Conjuntivas normocrômicas, anel bem posicionado, com discreto edema na incisão superior.

Em 08/10/98 (51 dias pós-op), estava assintomático, com o exame:

AV OE: $s c=20 / 50 \quad c c=20 / 40(-1,50 D)$

Em 13/04/99 (8 meses pós-op):

AV OE: $s c=20 / 60 \quad c c=20 / 30\left(-3,50-2,00 \times 120^{\circ}\right)$

\section{DISCUSSÃO}

Percebe-se, avaliando as figuras topográficas anteriores, um nítido aplainamento do ápice das córneas de aproximadamente de 6 dioptrias no caso 1 e de $10 \mathrm{D}$ no caso 2 . O astigmatismo corneano resultante também foi de pequena monta, não ultrapassando 4,00 dioptrias no caso 1 e 5,60D no caso 2. A acuidade visual vem mantendo-se boa até a última consulta em ambos os casos ( 3 meses pós-op no primeiro caso e 8 meses no segundo), sendo necessárias avaliações posteriores para observarmos sua evolução de longo prazo.

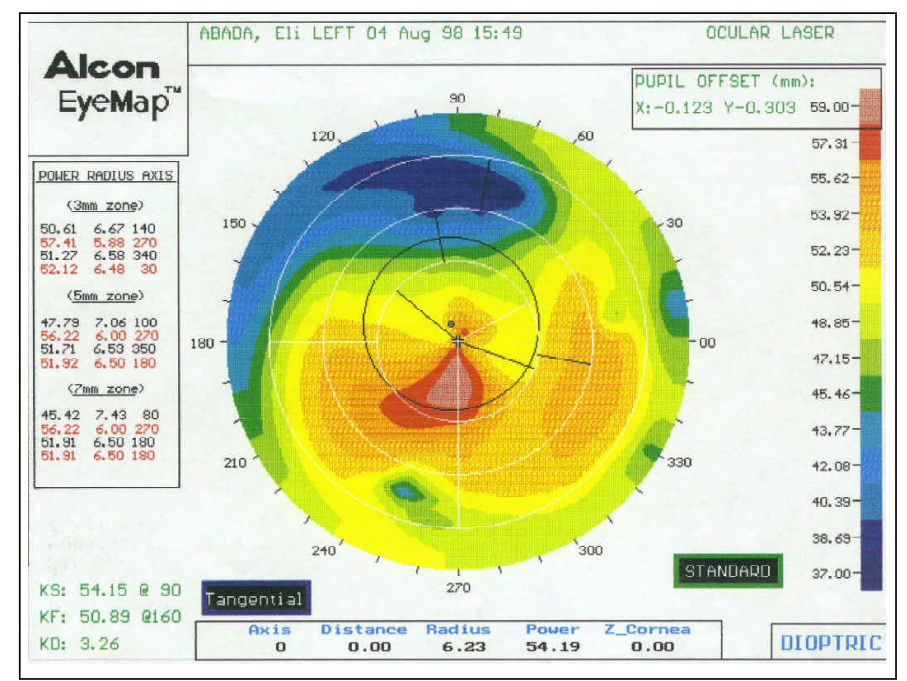

Fig. 3 - Aspecto topográfico do pré-operatório do caso 2 em 04/08/98.

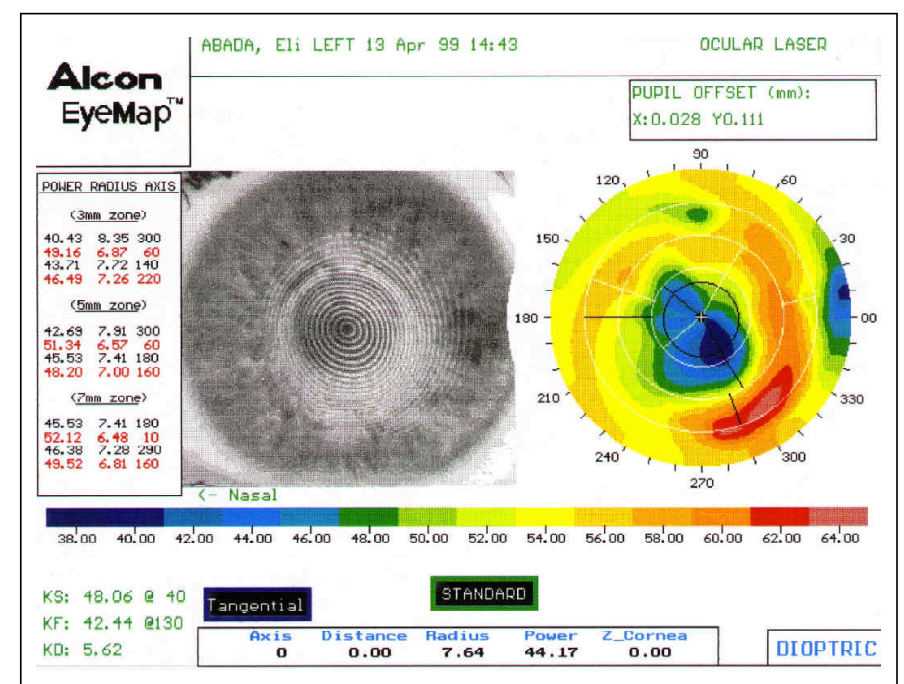

Fig. 4 - Aspecto topográfico do pós-operatório do caso 2 em 13/04/99.

A regressão de algumas dioptrias após a cirurgia da alta miopia com PRK é, até certo ponto, explicada por uma combinação entre hiperplasia epitelial e um rearranjo que ocorre na arquitetura do estroma ${ }^{4}$. Devemos excluir a possibilidade de estarmos diante de uma refração pré-operatória pouco cuidadosa, podendo envolver até algum distúrbio de acomodação. O problema se torna grave quando se trata de uma ablação excessiva da córnea, desestabilizando sua arquitetura e permitindo a sua ectasia. Como esta complicação tende a ser progressiva, a correção com óculos ou lentes de contato são apenas paliativas. Resta o transplante de córnea como último recurso para restabelecer as características ópticas do olho, mesmo sendo um procedimento de significativo risco, recuperação lenta e dependente das filas de doação. O mais importante é a prevenção e, para isso, temos alguns princípios de Machat para a manutenção da estabilidade corneana ${ }^{5}$ : 
- a profundidade da ablação não deve exceder a 50\% da espessura da córnea.

- a espessura total da córnea deve ser de 400 micra ou maior.

- a espessura do estroma residual deve ter no mínimo de 200 micra, embora o ideal é que seja pelo menos 250 micra.

Embora o uso de anéis intraestromais para correção de erros refrativos não seja um conceito novo ${ }^{1,2}$, a técnica, instrumental e próteses ainda estão sob investigação. $\mathrm{O}$ aplainamento corneano com o ACI já foi demostrado em outros trabalhos ${ }^{3}$, mas não encontramos nenhuma referência quanto à sua utilização em córneas ectásicas. Este procedimento refrativo ainda não é amplamente difundido no mundo para a correção do ceratocone, alta miopia e astigmatismo irregular.

A recuperação pós-cirúrgica é rápida, já se percebendo melhoras no dia seguinte, embora mudanças refrativas levem 3 meses para estabilizar. É comum que ocorra alguma fotofobia que pode durar algumas semanas, e visão de halos que podem durar aproximadamente 3 meses.

Os pacientes foram alertados sobre a natureza experimental da cirurgia e seus riscos inerentes.

\section{CONCLUSÕES}

Este estudo preliminar mostra que o implante intraestromal do Anel de Ferrara pode obter sucesso no tratamento da ectasia corneana pós PRK, aplainando sua área central e evitando sua progressão. Sendo assim, permite que se retarde ou até mesmo evite a evolução destes casos para um transplante de córnea com todos os seus riscos, lenta recuperação e dependência da agilidade das filas de doações.

\section{SUMMARY}

Purpose: To evaluate the action of Ferrara's Ring in the treatment of corneal ectasia after Excimer Laser.

Methods: Ferrara's Ring, that is already being applied in the treatment of keratoconus, high myopia and irregular astigmatism, has been used to stabilize and to correct the corneal ectasia of 2 patients submitted to PRK.

Results: After the surgical procedure, a flattening of the central cornea was observed, with approximately $6 \mathrm{D}$ (first case) and $10 \mathrm{D}$ in the second, with visual acuity improvement. The results have remained stable until this moment. The postoperative follow-up was of 3 and 8 months, respectively. Conclusions: Ferrara's ring has obtained success in the difficult treatment of this surgical complication, allowing delay of or even avoiding the evolution of these cases to a corneal transplantation with all its risks, slow recovery and dependence on the agility of the donation lines.

Keywords: Corneal ectasia; Refrative surgery; Ferrara's ring.

\section{REFERÊNCIAS BIBLIOGRÁFICAS}

1. Barraquer JL. Modification of refraction by means of intracorneal inclusion. Int Ophth Clin 1996;6:53.

2. Belau PG, Dyer JA, Ogle KN, Henderson JW. Correction of ametropia with intra-corneal lenses: An experimental study. Arch Ophthalmol 1964;72:541.

3. Neves RA, Nosé W, Belfort Jr. R, Burris TE, Schanzlin DJ. Intrastromal Corneal Ring. Arq Bras Oftalmol 1996;59:224.

4. Goodman GL, Trokel SL, Stark WJ. Corneal healing following laser refractive keratectomy. Arch Ophthalmol 1989;107:1799.

5. Machat JJ. PRK complications and their management. In: Excimer laser refractive surgery: practice and principles. Thorofare, Slack Incorporated, $1996 ; 169-210$ 